\title{
Ad hoc physical Hilbert spaces in Quantum Mechanics
}

\author{
Francisco M. Fernández and Javier Garcia \\ INIFTA (UNLP, CCT La Plata-CONICET), División Química Teórica, Blvd. 113 \\ S/N, Sucursal 4, Casilla de Correo 16, 1900 La Plata, Argentina \\ e-mail:fernande@quimica.unlp.edu.ar \\ and \\ Iveta Semorádová and Miloslav Znojil \\ Nuclear Physics Institute ASCR, \\ 25068 Rež, Czech Republic \\ e-mail: znojil@ujf.cas.cz
}

\begin{abstract}
The overall principles of what is now widely known as PT-symmetric quantum mechanics are listed, explained and illustrated via a few examples. In particular, models based on an elementary local interaction $\mathrm{V}(\mathrm{x})$ are discussed as motivated by the naturally emergent possibility of an efficient regularization of an otherwise unacceptable presence of a strongly singular repulsive core in the origin. The emphasis is put on the constructive aspects of the models. Besides the overall outline of the formalism we show how the low-lying energies of bound states may be found in closed form in certain dynamical regimes. Finally, once these energies are found real we explain that in spite of a manifest non-Hermiticity of the Hamiltonian the time-evolution of the system becomes unitary in a properly amended physical Hilbert space.
\end{abstract}




\section{Introduction}

\subsection{The methodical framework}

The scope and range of any study of mathematical structures as used in quantum theory strongly depend on the domain of prospective applications. In this sense we feel inspired, in particular, by the success of nuclear physicist's strategy of the so called interacting boson models as reviewed, say, in Ref. [1]. This review may be recalled as illustrating the efficiency of a judicious, nontrivial choice of the Hilbert space, due to which the states of a given quantum system may be represented in an optimal, computation-friendly manner.

In the concrete nuclear-physics phenomenological setting as presented in Ref. [1] the argument supporting the importance of availability of several alternative Hilbert spaces $\mathcal{H}$ yielding equivalent physical predictions may be made more specific. Indeed, it is well known that whenever we perceive an atomic nucleus in terms of its nucleonic (i.e., fermionic) degrees of freedom, it is just a routine task to recall the principle of classical-quantum correspondence and to postulate the existence of a "realistic" Hamiltonian in its widely accepted kinetic- plus potential-energy "microscopic" form of an operator $\mathfrak{h}=\mathfrak{h}^{(A)}=-\sum_{i}^{A} \triangle_{i}+\sum_{j} \sum_{k} V_{j k}$. This operator is defined and, by construction, safely self-adjoint in the traditional Hilbert space $\mathcal{H}^{(P)} \equiv L^{2}\left(\mathbb{R}^{3 A}\right)$ (here, using the notation as introduced in our review paper [2], the superscript ${ }^{(P)}$ stands for "primary" alias "physical").

In the framework of nuclear physics, unfortunately, the practical success of the use and study of the realistic Hamiltonians $\mathfrak{h}^{(A)}$ remained restricted just to the very light nuclei. For any heavier (and, in particular, heavy) atomic nucleus, the "brute-force" numerical diagonalization technique failed to provide a satisfactory precision of energies (in numerical context) and/or a satisfactory intuitive insight into the structure of wave functions (say, in the context of testing the predictions experimentally).

In a way reviewed in [1], a decisive and persuasive progress has been achieved after a replacement of the microscopic, fermionic Hamiltonians by their various effective (and, in principle at least, isospectral) partners $H=H^{(A)}=\Omega^{-1} \mathfrak{h}^{(A)} \Omega$. A particularly productive principle of construction of the latter effective Hamiltonians $H^{(A)}$ relied upon the intuitively appealing idea that due to certain specific (and more or less known) features of the "realistic" inter-nucleon forces $V_{j k}$, the pairs of fermions inside a nucleus may often be perceived as coupled, intuitively at least, into certain effective, bosons-resembling quasi-particles.

In the purely pragmatic context, an exceptional and by far the most successful 
implementation of the latter constructive recipe has been found in an appropriate adaptation of the Dyson's older idea [3] by which one makes an ansatz $\mathfrak{h}=\Omega H \Omega^{-1}$ while choosing the ad hoc "Dyson's map" $\Omega$ non-unitary. Thus, as long as this implies that

$$
\mathfrak{h}^{\dagger}=\left[\Omega^{-1}\right]^{\dagger} H^{\dagger} \Omega^{\dagger}=\Omega H \Omega^{-1}
$$

i.e.,

$$
H^{\dagger} \Omega^{\dagger} \Omega=\Omega^{\dagger} \Omega H
$$

one has to deal with a new, effective Hamiltonian which appears manifestly nonHermitian in all of the generic, non-trivial situations in which the superposition of the two Dyson's maps remains nontrivial, $\Omega^{\dagger} \Omega=\Theta \neq I$.

From the point of view of mathematics the latter unexpected observation did not in fact lead to any really serious complications. Indeed, people (including, and listed by, the authors of [1]) quickly imagined that although one has $H \neq H^{\dagger}$ in the "effective", bosonic Hilbert space $\mathcal{H}^{(F)}$ with the "usual" inner product (here, although the superscript ${ }^{(F)}$ stands for "friendly", this acronym may be also re-read as "false", due to the apparent violation of the unitarity of the evolution generated by the non-self-adjoint generator $H$ ), one may simply redefine the inner product using the "metric operator" $\Theta$. In this way one arrives at another, physical bosonic Hilbert space $\mathcal{H}^{(S)}$. The superscript ${ }^{(S)}$ stands here for "standard" because inside the new, unitarily non-equivalent bosonic Hilbert space $\mathcal{H}^{(S)}$, the evolution generated by the same generator alias Hamiltonian $H$ appears now, in full agreement with the standard textbooks on quantum theory, unitary.

We may summarize that the introduction of the "standard" Hilbert-space-metric operator $\Theta=\Omega^{\dagger} \Omega \neq I$ enables us to replace the original, correct but computationunfriendly physical representation space $\mathcal{H}^{(P)}$ by its unitarily equivalent amendment $\mathcal{H}^{(S)}$ (which may be called, deservedly, "sophisticated" [2]). The whole idea may be 
given the form of a diagram

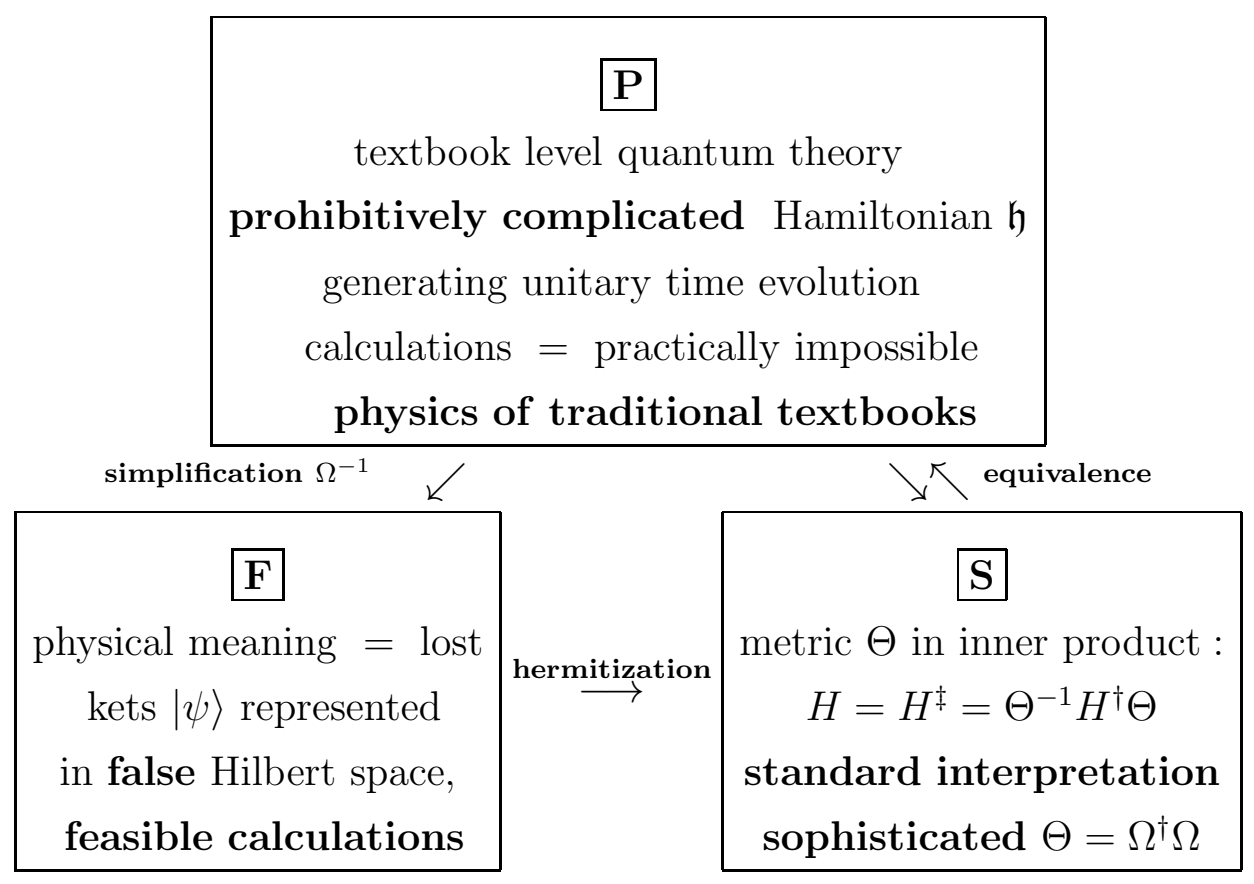

We see that for formal reasons it is recommended that the $P \leftrightarrow S$ unitary equivalence is realized in two steps. Firstly, the kets $\mid \psi \succ \in \mathcal{H}^{(P)}$ (notice their "curved" denotation as proposed in [2]) are interpreted as Dyson-map images $|\psi \succ=\Omega| \psi\rangle$ of their "bosonic" representants in an auxiliary, unphysical, "false", F-superscripted Hilbert space, $|\psi\rangle \in \mathcal{H}^{(F)}$. Secondly, a new inner product is introduced, in $\mathcal{H}^{(F)}$, just to define, formally, another, viz., the second physical Hilbert space $\mathcal{H}^{(S)}$.

The main advantage of the resulting representation of a given quantum system via a triplet of Hilbert spaces may be seen in the underlying implicit assumption of the thorough simplification $\mathfrak{h} \rightarrow H$ of the Hamiltonian paid by an affordable complication of the Hermitian conjugation in the physical Hilbert space. Indeed, we have to replace the traditional "transposition plus complexification" maps (viz., $\mathfrak{h} \rightarrow \mathfrak{h}^{\dagger}$ and $H \rightarrow H^{\dagger}$ ) in $\mathcal{H}^{(P)}$ and $\mathcal{H}^{(F)}$, respectively, by their more sophisticated, metric-dependent analogue $H \rightarrow H^{\ddagger}=\Theta^{-1} H^{\dagger} \Theta$ in $\mathcal{H}^{(S)}$.

\subsection{Non-Hermitian differential-operator Hamiltonians}

Naturally, the key assumption of the decisive simplicity of the "new" Hamiltonian $H$ has been successfully verified not only in the above-mentioned realistic context of the models of nuclei but also, say, for the first-quantized Klein-Gordon equation [4]. An exceptional methodical role has been played by the Buslaev's and Grecchi's "wrong-sign" anharmonic oscillator [5] where both of the Hamiltonian-operator representatives $H=H^{(B G)}$ and $\mathfrak{h}=\mathfrak{h}^{(B G)}$ preserved the elementary differential-operator 
form containing just a local interaction potential, viz.,

$$
H^{(B G)}=\frac{1}{2}\left(-\frac{d^{2}}{d y^{2}}+\frac{j^{2}-1}{4(y-i \varepsilon)^{2}}\right)-g^{2}(y-i \varepsilon)^{4}, \quad y \in \mathbb{R}
$$

and

$$
\mathfrak{h}^{(B G)}=-\frac{d^{2}}{d x^{2}}+(1-g x)^{2} x^{2}-\frac{1}{2}(2 g x-1), \quad x \in \mathbb{R}
$$

(see also [6]; incidentally, even the special $g=0$ case of this model proved worth a rediscovery [7]).

In the case of many other, generic complex local potentials entering the differential operators

$$
H=-\frac{d^{2}}{d y^{2}}+V(y), \quad y \in \mathbb{R}
$$

many authors revealed that the spectrum may still remain real, discrete and bounded from below [8, 9, 10]. In other words, every demonstration that a mathematically tractable operator (5) possesses such a spectrum opens the possibility of assigning, to it, the status of an observable (i.e., e.g., of a Hamiltonian) of a hypothetical quantum system, i.e., the status of a self-adjoint operator in an ad hoc physical Hilbert space $\mathcal{H}^{(S)}$.

Several theoretical as well as practical challenges emerge. Even in the context of pure mathematics one quickly reveals that any - even approximate - construction of the metric $\Theta$ is by far not easy [11]. The difficulties of mathematical nature are accompanied by their phenomenological parallels. The most important one lies in the generic loss of the locality of the operator $\Theta$ which is reflected by the loss of the observability of the coordinate. This may have a destructive impact upon the traditional "kinetic plus potential energy" tractability of Hamiltonians (5). Their "point-particle" interpretation may get lost [12], certain "no-go" theorems emerge in the context of scattering [13], etc.

Amazing as it may seem, all of these difficulties may prove more than compensated by the perspective of innovations (in this context, Refs. [14, 15] offer a useful reading). An encouragement of realistic quantum model-building may be sought also in the flexibility of the phenomenologically motivated choice of non-trivial metrics. For illustration one may recall Ref. [4] where the old problem of proper quantummechanical interpretation of Klein-Gordon equation (describing, e.g., the physics of pionic atoms) has been resolved via the use of $\Theta \neq I$.

In our present paper we intend to point out that the quantum theory in its threeHilbert-space-representation (THSR) form may find one of its fairly interesting new illustrations and applications in the apparently traditional context of Eq. (5) where 
one would merely add the new assumption that the potential $V(y)$ itself is singular and, hence, not well defined along the real line of $y$. This is, in fact, the situation which was assumed in our recent paper [16] where we felt inspired by the toy model (3) of Ref. [5] and where we studied Schrödinger equation

$$
\left[-\frac{d^{2}}{d s^{2}}+U(s)\right] \phi_{n}(s)=E_{n} \phi_{n}(s), \quad U(s) \equiv V[x(s)]=(s-\mathrm{i} \varepsilon)^{2}+\frac{g^{2}}{(s-\mathrm{i} \varepsilon)^{6}}
$$

in which $s \in \mathbb{R}$, i.e., in which one had to assume that $y \notin \mathbb{R}$ in the context of Eq. (5).

In Ref. [16] we emphasized that the obligatory starting point of the applications of the THSR formalism should be seen in the demonstration of the reality of the spectrum. For the present review-paper purposes these considerations will be briefly summarized in section 3. In subsequent section 4 we shall then extend the class of potentials as well as the scope of the method. We shall complement the large-g approximate constructions of section 3 by a systematic higher-order perturbationexpansion technique. This will enable us to study the singular models at finite couplings $g \ll \infty$.

Being well aware of the fact that our perturbation series may have (and probably do have [17]) a vanishing radius of convergence, our perturbation-series description of the systems with finite couplings will be complemented and paralleled (i.e., tested, in section 5) by an independent numerical reconstruction of the spectrum using the so called Riccati-Padé method [18]. We shall reveal that for a number of specific choices of strongly singular potentials, extremely small error bars may be reached by our perturbation-series estimates.

A successful confirmation of the applicability of the two independent methods to a new class of quantum models in a fairly nonstandard domain of their implementation will be obtained. Still, our main message will lie elsewhere. Our numerically obtained values of the low lying bound-state energies will be re-read as not possessing, within error bars, any imaginary components. In other words, a "spectrum-reality" confirmation will be declared covering the dynamics beyond the currently published area of not too singular potentials.

Our present observations will finally be discussed in section 6. Naturally, our study of singular potentials still leaves multiple open questions unanswered. It must be emphasized, nevertheless, that whenever available, the better, rigorous mathematical proofs of the reality of spectra of non-Hermitian Hamiltonians (5) appeared almost prohibitively complicated even for regular potentials [10, 19]. 


\section{The key problem: the reality of the spectrum}

Let us reemphasize that in our present class of models with complex "coordinates" $y$ and "potentials" $V(y)$ the conventional self-adjoint nature of the Hamiltonian is certainly lost in the "false" space $\mathcal{H}^{(F)}$. Still, whenever one proves that the resulting spectrum of energies is real and discrete and bounded from below, the way is open towards the reconstruction of the appropriate Hilbert space $\mathcal{H}^{(S)}$ in which our Hamiltonian becomes self-adjoint. Let us now recall, for illustration purposes, a few most elementary examples.

\subsection{Radial regular examples}

In 2001, Dorey, Dunning and Tateo [10] considered various complex potentials $V(y)$ in Schrödinger equations and they attracted the reader's attention, in particular, to the weakly singular centrifugal-like components $V^{(D D T)}(y)=\ell(\ell+1) / y^{2}$ of the interactions. As long as the threshold behavior of the general wavefunctions then remained easily tractable in closed form,

$$
\psi(y) \sim c_{1} y^{\ell+1}+c_{2} y^{-\ell}, \quad|y| \ll 1,
$$

these authors were allowed to ignore the singularity whenever they restricted the "coordinate" $y$ to the lower half of the complex plane, $\operatorname{Im} y=-\varepsilon<0$.

Now we see the difference. For the real $y$ one would only be allowed to work on a half-axis, with $y \in(0, \infty)$. For the complex $y=s-\mathrm{i} \varepsilon$ the boundary conditions must necessarily be changed - while one omits the redundant boundary condition in the origin $y=0$, a new constraint emerges as the left asymptotic boundary condition enters the scene at $s \rightarrow-\infty$. Naturally, not only physics (i.e., the interpretation of measurements) but also mathematics (i.e., typically, spectra - see, for example, [7]) get changed.

\section{$2.2 \quad$ A wrong-sign quartic example}

Buslaev and Grecchi [5] were probably the first who demonstrated, constructively, that the Dyson's isospectral mapping $\Omega$ between Hamiltonians may cause a truly thorough change of the Hilbert space. In loc. cit. they constructed the mapping between Hamiltonians (3) and (44) and showed that in the former operator the change of the parameter $\varepsilon$ does not change the spectrum at all. In the light of the analyticity properties of the potentials such an observation is not too surprising. At the same 
time, in the context of Ref. [16] the same freedom of the choice of $\varepsilon$ (which, in fact, meant the freedom of a parallel shift of the complex line of $y$ in Schrödinger equation) proved to be of fundamental importance.

Any generalization of the Buslaev's and Grecchi's results to a less exceptional potential acquires, as a rule, the form of an approximate construction. In this spirit, the new and particularly challenging strongly singular and $\mathcal{P} \mathcal{T}$-symmetrically regularized inverse-sextic-repulsion model of Eq. (6) will be now considered as a methodical guide as well as one of the most natural candidates for an apparently non-Hermitian (in the "false" space $\mathcal{H}^{(F)}$ ) but (in the "standard" space $\mathcal{H}^{(S)}$ ) still unitarily evolving quantum model.

\section{$3 \quad$ Strongly repulsive potentials}

The reasons of our interest in the strongly singular and complex-shift regularized quantum models are explained in Appendix A below. Our discovery of their mathematical appeal dates back to Ref. [20] where their perturbative tractability has been revealed and tested. Perturbation expansions were found to work there for a broad complex-valued subfamily of regular potentials. In this sense, it will only be necessary to demonstrate here that the presence of the strongly repulsive barriers need not obstruct the applicability of the same large $-g$ perturbation-expansion techniques.

\subsection{Schrödinger equations at special values of $\varepsilon$}

The main weak point of the rather universal large $-g$ perturbation-expansion technique as explained, e.g., in Ref. [17] is that its convergence to exact results cannot be guaranteed in general. One only has to use the formalism as a source of suitable asymptotic series and approximants. In this sense, such a perturbation recipe will still satisfy our present needs sufficiently well.

The essence of the formalism lies in several assumptions. Firstly, one must require that in the regime of large couplings $g$ the potential develops a pronounced minimum with a negligible imaginary component. Thus, the first derivative of the potential function must vanish at a certain complex value of the coordinate $x=R_{m}$. One writes $V^{\prime}\left(R_{m}\right)=0$ and treats such a formula as an elementary algebraic equation determining, implicitly, the unknown eligible complex minima $R_{m}$. For our model of Eq. (6), in particular, the latter equation reads $2 R_{m}^{8}=6 g^{2}$ and leads to the eight 
well-separated closed-form candidates for the minimum,

$$
R_{m}=R e^{\mathrm{i} \pi(m-1) / 4}, \quad R=\left|3^{1 / 8} g^{1 / 4}\right| \gg 1, \quad m=1,2, \ldots, 8
$$

In order to obtain an efficient approximation recipe we need to require the positivity of the second derivative of our potential at its stationary points. Such a necessary condition is restrictive and not too easily satisfied. Fortunately, the verification yields the same real quantity at all $m$ for our toy model,

$$
V^{\prime \prime}\left(R_{m}\right)=2+42 \frac{g^{2}}{R_{m}^{8}}=2+42 \frac{g^{2}}{3 g^{2}}=16 .
$$

In a systematic analysis we reveal that the uppermost root $R_{3}$ lies on the cut. A hardly solvable regularized double-well Schrödinger equation is also obtained if one selects the complex line of its integration as intersecting the pairs of stationary points $R_{2}$ and $R_{4}$ or $R_{1}$ and $R_{5}$ or $R_{8}$ and $R_{6}$.

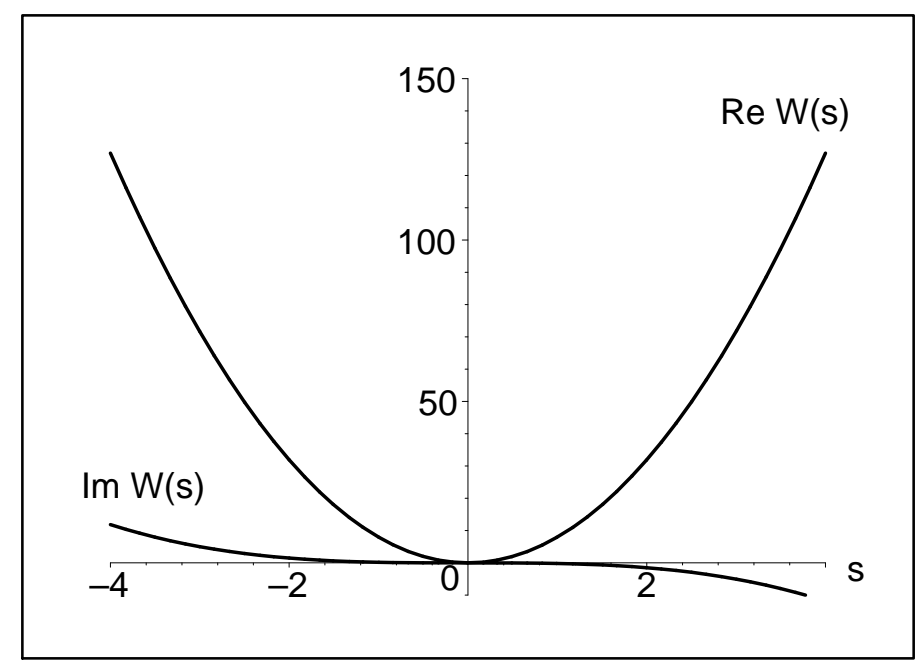

Figure 1: The real and imaginary parts of the upwards-shifted potential function of Eq. (7), with $W(s)=4 R^{2} / 3+V[x(s)]$ and $\varepsilon=R=100$.

The last and only eligible candidate for a "useful" stationary point is the purely imaginary root $R_{7}$ which is, in this sense, unique. We come to the conclusion that once we let the complex line $x(s)$ cross the complex point $R_{7}$ (it is easy to show that this means that we choose $\varepsilon=R$ ) we have satisfied all requirements. Consequently, along the line $x(s)$ the real part of our potential is really characterized by a pronounced minimum while its imaginary component becomes negligible in the regime of large parameters $R$ (cf. Fig. 11). Hence, we may try to apply the recipe of Ref. [20]. Let us now describe the results in full detail. 


\subsection{Harmonic-oscillator approximation}

Let us recall our potential,

$$
V(x)=x^{2}+\frac{R^{8}}{3 x^{6}}
$$

At the large real $R$ and at the small complex shifts $\mathrm{i} \varepsilon=s-x(s)$ the shape and $s$-dependence of function (7) remains dominated by its singularity. For the large $\varepsilon \sim R$ this domination gets suppressed. The complex function $V[x(s)]$ acquires a deep minimum at $x=-\mathrm{i} R$ (cf. Fig. 1) so that we may try to Taylor-expand the potential near the point $R_{7} \equiv-\mathrm{i} R$,

$$
\begin{gathered}
V[x(s)]=-\frac{4}{3} R^{2}+8 s^{2}-\mathrm{i} \frac{56}{3 R} s^{3}-\frac{42}{R^{2}} s^{4}+\mathrm{i} \frac{84}{R^{3}} s^{5}+\frac{154}{R^{4}} s^{6}- \\
-\mathrm{i} \frac{264}{R^{5}} s^{7}-\frac{429}{R^{6}} s^{8}+\mathrm{i} \frac{2002}{3 R^{7}} s^{9}+\frac{1001}{R^{8}} s^{10}-\ldots
\end{gathered}
$$

The decrease of the higher-order terms appears so quick that the harmonic-oscillator term is dominant and that the radius of convergence of the series remains large, equal to $R$. The most important observation is that along the line of the integration of the equation, the imaginary components of the potential become entirely negligible.

The polynomial truncations of series (8) remind us of the popular complex powerlaw interaction models (cf. Ref. [14]). Once we restrict our attention just to the first two terms of series Eq. (8) we even arrive at the exactly solvable model of the usual, real harmonic oscillator. Its low-lying spectrum of bound states is well known yielding the fairly reliable approximation

$$
E_{n}=-\frac{4 R^{2}}{3}+(2 n+1) \sqrt{8}+\mathcal{O}\left(\frac{1}{R}\right), \quad n=0,1, \ldots .
$$

All of the higher-order contributions lead just to asymptotically vanishing corrections to the energies. Thus, the first few orders of perturbation theory lead to the low-lying energy levels which are all equidistant, real and negative.

We may summarize that the main message delivered by this section is that under the assumption that the real coupling constant $g$ is kept very large, the low lying spectrum of energies can be found, in spite of the manifest non-Hermiticity of Hamiltonian $H_{1}^{(I S)}$, real. One may expect that the Hamiltonian may be reinterpreted again as self-adjoint in an ad hoc space $\mathcal{H}^{(S)}$.

\section{Systematic perturbation expansions}

Our considerations of preceding section were restricted to large $g \gg 1$. After the publication of the first results of this type via arXiv [16] our present team of authors 
has been established to analyze the possibilities of an extension of these observations to the less fictitious dynamical regime of smaller, finite couplings $g<\infty$ in the same potential as well as to some other, more general singular potentials, say, of the two-term form

$$
V(x)=-(\mathrm{i} x)^{2+\alpha}-\frac{g^{2}}{(\mathrm{i} x)^{6+\beta}} .
$$

Each of these functions may enter the ordinary differential Schrödinger equation subject to the same replacement $x \rightarrow x(s)=s-\mathrm{i} \varepsilon$ as above.

\subsection{The case of real potentials as a methodical guide}

Hamiltonian operators of the usual self-adjoint form (where $V(x)$ is real, non-singular and confining) admit the approximate determination of the low lying spectrum of bound states via a localization of the real minimum $x_{0} \in \mathbb{R}$ of the potential, i.e., via a determination of the root $x_{0}$ of a transcendental algebraic equation $V^{\prime}\left(x_{0}\right)=0$ under constraint $V^{\prime \prime}\left(x_{0}\right)>0$ [17]. In such a case one can also amend the approximation using perturbation theory. After a change of variables $x=x_{0}+\beta s$ where $-\infty<$ $s<\infty$ and where $\beta$ is an arbitrary auxiliary real scaling factor, one may expand $V\left(x_{0}+\beta s\right)$ in Taylor series near $s=0$,

$$
V\left(x_{0}+\beta s\right)=\sum_{j=0}^{\infty} V_{j} \beta^{j} s^{j} .
$$

In the new Hamiltonian operator

$$
H=\beta^{-2}\left(-\frac{d^{2}}{d s^{2}}+V_{2} \beta^{4} s^{2}+\sum_{j=3}^{\infty} V_{j} \beta^{j+2} s^{j}\right)
$$

we choose $\beta=V_{2}^{-1 / 4}$ so that

$$
H=\sqrt{V_{2}}\left(-\frac{d^{2}}{d s^{2}}+s^{2}+\sum_{j=1}^{\infty} \frac{V_{j+2}}{V_{2}} \beta^{j} s^{j+2}\right) .
$$

Now one decides to apply perturbation theory to Schrödinger equation

$$
\frac{1}{\sqrt{V_{2}}} H \psi=\epsilon \psi
$$

and one obtains the usual perturbation series for the eigenvalues

$$
\epsilon=\sum_{j=0}^{\infty} \epsilon_{j} \beta^{j}
$$


By construction one has $\epsilon_{0}=\epsilon_{0}(v)=2 v+1, v=0,1, \ldots$. Since the transformation $(\beta, s) \rightarrow(-\beta,-s)$ leaves the Hamiltonian invariant we may conclude that $\epsilon_{2 j+1}=0$ at all $j=0,1, \ldots$.

In the above context the main mathematical idea lying beyond the considerations of Ref. [16] (cf. also section 3 above) is that all of the main components of the above construction may remain applicable even if one leaves the real axis of $x$ and if one performs an analytic continuation of potential $V(x)$ into the complex plane of $x$. The only news is that in the complex case, the potential need not have the required real-harmonic-oscillator minima at all. Vice versa, the existence of these very specific minima (as shown, constructively, above) should be perceived as a very specific feature of certain "privileged" potentials. In other words, not all potentials would prove tractable by the present method.

\subsection{Perturbation expansions near a complex minimum $x_{0}$}

Let us now return to our family of potentials of Eq. (10) and let us try to determine all of its complex stationary points from the vanishing-derivative condition $V^{\prime}=0$. For methodical purposes we may just contemplate the small exponents $\alpha$ and $\beta$. We find out that the eligible negative imaginary stationary point $x_{0}$ as obtained at $\alpha=\beta=0$ (i.e., the complex coordinate $x_{0}=R_{7}=-\mathrm{i} T$ in the notation of Ref. [16]) merely moves to an amended $\alpha \neq 0 \neq \beta$ candidate for the minimum $x_{\text {min }}=-\mathrm{i} T$ where the real quantity $T$ is such that (say, at positive $\alpha$ and $\beta$ )

$$
T^{8+\alpha+\beta}=g^{2} \frac{6+\beta}{2+\alpha} .
$$

At this stationary point we also evaluate the second derivative exactly,

$$
V^{\prime \prime}\left(x_{\min }\right)=(2+\alpha) T^{\alpha}(8+\alpha+\beta) .
$$

This number is real and positive so that the assumptions of the applicability of the method of paragraph 4.1 are satisfied. The imaginary part of the potential remains negligible and the leading-order harmonic-oscillator approximation of Ref. [16] will keep working.

We are now prepared to Taylor-expand the potential. Besides the zero-order term

$$
-\frac{T^{2+\alpha}(8+\alpha+\beta)}{6+\beta}
$$

and besides the vanishing first order term we evaluate easily also the second order term

$$
1 / 2\left(2 \beta+\alpha \beta+16+10 \alpha+\alpha^{2}\right) T^{\alpha}
$$


The not too exciting news are coming with the third-order correction

$$
1 / 6 i(2+\alpha) T^{-1+\alpha}\left(\alpha+\alpha^{2}-56-15 \beta-\beta^{2}\right)
$$

where, incidentally, the last bracket factorizes, $(8+\alpha+\beta)(\alpha-7-\beta)$. Finally, the fourth-order part of the potential reads

$$
\begin{gathered}
-1 / 24\left(2 \beta^{3}+\alpha \beta^{3}+24 \alpha \beta^{2}+48 \beta^{2}+\right. \\
\left.+191 \alpha \beta+382 \beta+502 \alpha-\alpha^{2}+2 \alpha^{3}+\alpha^{4}+1008\right) T^{-2+\alpha}
\end{gathered}
$$

and may be simplified as well. Indeed, the bracket factorizes again,

$$
(2+\alpha)(8+\alpha+\beta)\left(\alpha^{2}-8 \alpha-\alpha \beta+63+16 \beta+\beta^{2}\right) .
$$

The long factor still factorizes over

$$
\alpha_{ \pm}=4+1 / 2 \beta \pm 1 / 2 \sqrt{-188-48 \beta-3 \beta^{2}}
$$

where the discriminant will vanish at the real roots $\beta=-8 \pm 2 / \sqrt{3}$. One may also add that the alternative factorization over

$$
\beta_{ \pm}=1 / 2 \alpha-8 \pm 1 / 2 \sqrt{-3 \alpha^{2}+4}
$$

seems simpler.

Once one moves to the higher orders of perturbation series, an explicit display of formulae would become clumsy and counterproductive. Still, it is possible to store the formulae in the computer and use them just for an evaluation of numerical predictions.

The computer-supported analysis and numerical tests of these results will be shown to enable us to conclude that the discussion as given in Ref. [16] remains applicable also to the more complicated potentials. The inclusion of the new parameters $\alpha$ and $\beta$ does not change the overall qualitative picture of the spectra. In what follows, we shall need just a routine procedure for obtaining the perturbation series approximation up to the $M$-th term, yielding the approximate energy values $E_{n}^{(M)}$ of the $n$-th bound state in broad intervals of finite couplings $g$.

\subsection{Sample choices of the integer values of $\alpha$ and $\beta$}

We will extend the results of Ref. [16] in two ways. Firstly we shall perform explicit calculations while restricting our attention to certain special integer values of $\alpha$ and $\beta$, considering the family of singular potentials

$$
V(x)=x^{2 m}+\frac{\lambda}{x^{2 n}}, \quad \lambda=\frac{m R^{2(m+n)}}{n}, \quad m, n=1,3, \ldots
$$


In the spirit of preceding paragraph 4.2 we redefined the coupling, $g \rightarrow R(g)$, and choose $m$ and $n$ as positive integers.

Formally, we may now apply perturbation theory up to arbitrarily large order. In this manner we obtain numerical results which may be compared with some other, nonperturbatively obtained values of bound state energies (cf. section 5 below). Technically, the construction of the perturbation series will be facilitated by the friendlier notation of Eq. (16).

First of all we notice that if $V(-x)=V(x)$ and if $a$ and $s$ are real, then $U(s)=$ $V(i a+s)$ has the property $U(-s)^{*}=V(-i a-s)=V(i a+s)=U(s)$ called, usually, $\mathcal{P} \mathcal{T}$-symmetry. Once we consider just the family of spiked oscillators (16) where $\lambda>0$ and where $m$ and $n$ are positive integers, the first minimality condition $V^{\prime}\left(x_{0}\right)=0$ yields

$$
x_{0}=\left(\frac{\lambda n}{m}\right)^{1 /[2(m+n)]} e^{\mathrm{i} \pi k /(m+n)}, \quad k=0,1, \ldots, 2(m+n)-1 .
$$

The root $x_{0}$ remains purely imaginary if we require that $m+n$ is even. This choice may simplify the discussion and it will be preferred in what follows. It also implies that for the second derivative we have

$$
V^{\prime \prime}\left(x_{0}\right)=4 m(m+n) x_{0}^{2(m-1)} .
$$

We see that $V^{\prime \prime}\left(x_{0}\right)>0$ only if $m$ is odd. Thus, whenever we want to keep our present discussion fully analogous to the one of Ref. [16], the value of $n$ should be also chosen odd.

\section{The numerical determination of the energies}

\subsection{Quadratically convergent numerical method}

For our present purposes it is important that the quickly convergent Riccati-Padé method (RPM, [18]) of the numerical determination of the eigenvalues of Hamiltonians is well adapted also to the treatment of the present, spatially asymmetric and complex potentials.

The key idea of the method is that one considers a correct wave function together with its logarithmic derivative

$$
f(x)=-\frac{\psi^{\prime}(x)}{\psi(x)}
$$


which, obviously, satisfies Riccati equation

$$
f^{\prime}(x)-f(x)^{2}+V(x)-E=0
$$

Once we Taylor-expand

$$
f(x)=\sum_{j=0}^{\infty} f_{j}\left(x-x_{0}\right)^{j}
$$

and substitute (21) into (20) we obtain the coefficients $f_{j}$ in terms of the two unknowns $E$ and $f_{0}=-\psi^{\prime}\left(x_{0}\right) / \psi\left(x_{0}\right)$. Next, splitting the sequence of coefficients into two subsequences,

$$
f_{e, j}=f_{2 j}, \quad f_{o, j}=f_{2 j+1}, j=0,1, \ldots
$$

we obtain both $E$ and $f_{0}$ as the roots of the system of the two coupled nonlinear algebraic equations

$$
\begin{aligned}
& H_{D e}^{d}\left(E, f_{0}\right)=\left|f_{e, i+j+d-1}\right|_{i, j=1}^{D}=0 \\
& H_{D o}^{d}\left(E, f_{0}\right)=\left|f_{o, i+j+d-1}\right|_{i, j=1}^{D}=0
\end{aligned}
$$

(cf. Refs. [18] for more details).

\subsection{Centrifugal-like spikes, $n=1$}

Before one applies the RPM numerical technique to singular models it seems useful to test the approach on a minimally singular example with $n=1$. Conveniently, we may then introduce the slightly modified, regularized logarithmic derivative

$$
f(x)=\frac{\sigma}{x}-\frac{\psi^{\prime}(x)}{\psi(x)} .
$$

With $\sigma=\left(1 \pm \sqrt{4 R^{4}+1}\right) / 2$ this choice exactly removes the pole of $\psi^{\prime}(x) / \psi(x)$ at the origin. In this case $f(x)$ satisfies the modified Riccati equation

$$
f^{\prime}+\frac{2 \sigma}{x}=f(x)^{2}+E-x^{2 m}
$$

With the ansatz

$$
f(x)=x \sum_{j=0}^{\infty} f_{j}(E) x^{2 j}
$$

we obtain the accurate eigenvalues in the form of the roots of the modified Hankel determinants $H_{D}^{d}(E)=\left|f_{i+j+d+1}\right|_{i, j=1}^{D}$. For sufficiently large $D$ we may, typically, choose $d=0$. In this manner the application of the RPM philosophy becomes more efficient than in the generic case. 
Table 1: The lowest RPM (i.e., exact) eigenvalue for several spiked oscillators

\begin{tabular}{||c|c|c||}
\hline \hline$R$ & $m=n=1$ & $m=1, n=3$ \\
\hline 20 & -798.00062499975585957 & -530.50539390089880261 \\
10 & -198.00249998437519531 & -130.50687623309973953 \\
5 & -48.009999000199950014 & -30.513112355071711530 \\
2 & -6.0622577482985496524 & -2.5774188753856708289 \\
1.5 & -2.6097722286464436550 & -0.35218352259563351294 \\
1 & -0.23606797749978969641 & 0.848803366333102053806 \\
\hline & $m=3, n=1$ & $m=n=3$ \\
\hline 20 & -255998040.41035854784 & -127997600.00562501215 \\
10 & -3999510.1106623371002 & -1999400.0225007778344 \\
5 & -62377.559943373182961 & -31100.090049835809532 \\
2 & -236.61574750381748921 & -104.5752232235887004 \\
1.5 & -34.899091771582975476 & -10.358453053216073663 \\
1 & -12.250254250322260411 & 1.52979838806531408256 \\
\hline \hline
\end{tabular}

\subsection{The test of the large-order perturbation results}

As long as we restricted our quantitative analysis to the subfamily (16) of potentials (10), we may proceed in the closest parallel with section 3. In particular, we may again put $x_{0}=-i R$, i.e., choose the same, very special and "user-friendly" distance $\varepsilon=R$ from the real line.

Beyond the leading-order-approximation framework as accepted in Ref. [16] we may now compare the exact numerical RPM predictions with those given by the semi-analytic perturbation expansions. In our present generalized, two-parametric models the function $U(x)=V(x-i R)$ still does not exhibit any singularities and it still possesses just a single global and pronounced minimum at $x_{0}$. Asymptotically this function is smooth and behaves as the power $x^{2 m}$ when $|x| \rightarrow \infty$.

This implies that a priori we may expect, in general, a good performance of perturbation theory. In order to test this expectation we decided to carry out the respective calculations for several lowest eigenvalues and for the decreasing sequence of couplings $R=20,10,2,1.5$ and 1 .

For illustration the "exact" RPM results for ground states are displayed first, in Table 1. The Table shows the lowest eigenvalue obtained by means of the RPM technique for several values of exponents $m$ and $n$ as discussed in paragraph 4.3 above. 


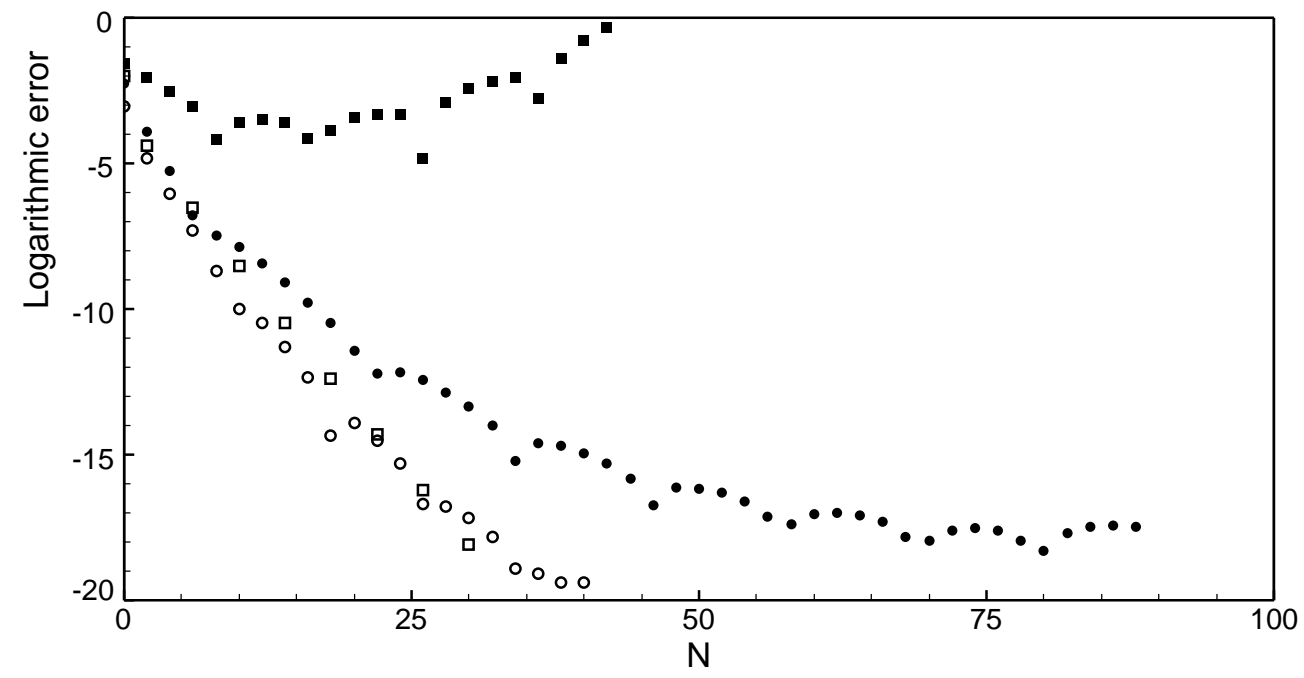

Figure 2: Logarithmic error $\log \left|\left(E^{[N]}-E^{R P M}\right) / E^{R P M}\right|$ for the ground state with $R=2$ for the oscillators given by $m=n=1$ (squares), $m=1, n=3$ (filled squares), $m=3, n=1$ (circles) and $m=n=3$ (filled circles)

Our subsequent tests of the performance of the perturbation series (i.e., of the precision of the $N$-th order perturbative ground-state approximants $E^{[N]}$ ) were based on the direct comparison with the RPM-based numerical results (of any prescribed precision). A sufficiently representative sample of these comparisons is summarized in Fig. 2. We used there a characteristic "intermediate" coupling constant $R=2$ and the same values of the exponents $m$ and $n$ as in Table 1 above.

The picture demonstrates, in general, the good performance of the partial sums $E^{[N]}$ of order $N$. We see that the logarithmic error of the perturbation series $\log \left|\left(E^{[N]}-E^{R P M}\right) / E^{R P M}\right|$ decreases, in most cases, in a sufficiently long interval of the perturbation order $N$. Still, the individual forms of the interaction carry certain specific characteristics.

\subsubsection{Potential $V(x)=x^{2}+\lambda / x^{2}$.}

At $m=n=1$ our approach leads to simplifications because $\epsilon_{4 j}=0, j=1,2, \ldots$. As expected the rate of convergence of perturbation series decreases with $R$. It even appears to converge at the coupling as small as $R=1$. On the other hand, the RPM numerical technique yields 20 accurate digits with $D=2(d=0)$ disregarding the value of $R$. Incidentally, the $m=n=1$ eigenvalue problem (i.e., the $\mathcal{P} \mathcal{T}$ symmetric 
harmonic oscillator of Ref. [7]) is exactly solvable so that we may be sure that both our methods yield approximate results that converge towards the exact ground-state energy $E_{00}=2-\sqrt{4 R^{4}+1}$.

\subsubsection{Potential $V(x)=x^{2}+\lambda / x^{6}$ of section 3, i.e., $m=1, n=3$.}

In this case perturbation expansion starts to oscillate, considerably, at a not yet too small value of $R=2$. The RPM appears to converge for all $R$ but the rate of its convergence decreases with the decreasse of $R$ rather quickly. One could say that among our quadruplet of toy models the oldest model of Ref. [16] seems least open towards perturbation-expansion amendments.

\subsubsection{Potential $V(x)=x^{6}+\lambda / x^{2}$, i.e., $m=3, n=1$.}

Perturbation expansions start to oscillate at smaller $R=1.5$ and they are, at all $R$, more stable than in the preceding example. Incidentally, also the rate of the convergence of the purely numerical RPM results is perceivably higher than in the preceding case.

\subsubsection{Potential $V(x)=x^{6}+\lambda / x^{6}$, i.e., $m=n=3$.}

In picture 2 we see that in spite of certain growth of complexity of the underlying formulae the numerical rate of convergence of the perturbation-theory approximations remains very satisfactory and does not exhibit oscillations even at the very high orders. Empirically one may reveal a certain apparent regularity (or periodicity in a monotonic decrease of perturbation-series errors) but no immediate explanation of this phenomenon seems available to us at present.

\section{Summary}

The recent extension of quantum theory to Hamiltonians $H=-d^{2} / d x^{2}+V(x) \neq H^{\dagger}$ where the coordinate is not observable, $x \notin \mathbb{R}$, was reviewed here and tested in an innovative context of the strongly singular potentials, say, of the form $V(x)=$ $(i x)^{\text {const }} x^{2}+g^{2} /\left[(i x)^{\text {const }} x^{2}\right]$. Three standard (viz., large- $g$, perturbation-expansion and numerical Riccatti-Padé) construction techniques were shown applicable to such a class of models. All of these methods were shown to yield mutually compatible results supporting the hypothesis of reality of the energies of the low-lying bound 
states, i.e., of a potentially self-adjoint nature of the Hamiltonian in a properly chosen ad hoc physical Hilbert space $\mathcal{H}^{(S)}$ with a nontrivial metric $\Theta \neq I$.

Originally, a similar, purely empirical observation of the possible reality of energies of bound states in a repulsive singular $\mathcal{P} \mathcal{T}$ symmetric potential was made, in Ref. [16], in a very restricted dynamical regime of very large repulsion strength $g \rightarrow \infty$. In our present continuation of this study we proceeded to the sub-asymptotic dynamical regime of finite couplings $g<\infty$ alias $R<\infty$.

What is shared here with Ref. [16] is the explicit clarification of the highly nontrivial physical non-equivalence and of a deep phenomenological contrast between the traditional, real-half-axis choice of the radial coordinate $r$ and the innovative, $\mathcal{P} \mathcal{T}$-symmetry-inspired complex (i.e., anomalous, unobservable) choice of the line of integration of the seemingly not too much different Schrödinger equation. In fact, the two complementary parts of an entirely new field of research in quantum theory are encountered. This fact may be perceived as a welcome and encouraging extension of possibilities of the model-building in quantum mechanics.

One should also mention that in the future analyses of singular models our deeper understanding of the possible underlying physics will require a further extension of the construction, in particular, towards some (i.e., at least, leading-order-form) metrics which would define the above-mentioned "Hermitizing" inner products in Hilbert space $\mathcal{H}^{(S)}$. What one could find encouraging also in this context is, for the present particular choice of interaction models, the large-coupling negligibility of the imaginary part of the potentials near their minima at $x_{0}$.

It is necessary to add that the subject itself is by far not exhausted. First of all, our present, RPM-based demonstration of the absence of the imaginary parts in energies is more or less purely numerical. Secondly, the perturbation method we tested remains restricted just to the low-lying part of the spectrum of energies. Even its compatibility with independent numerical RPM results does not offer a rigorous proof of course. A priori, within our present methodical framework one still cannot exclude the possibility of the presence of some exponentially small imaginary components in the energies.

This being said, our non-rigorous numerical results may still be declared important because their existence strengthens the intuitively sound persuasion that the time evolution of the underlying quantum systems cannot deviate from unitarity too much. In other words, one may expect that on a pragmatic and approximative level it is possible to consider our user-friendly new Hamiltonians as operators with a good chance of being perceived also as $\mathcal{P} \mathcal{T}$-symmetric and self-adjoint with respect 
to a suitable, nontrivial physical inner product in the underlying Hilbert space of admissible quantum states.

On the background provided by our previous paper [16] let us add that what is also provided by the present new models, methods and calculations is a long-expected extension of the leading-order approximations towards a systematic formalism of full-fledged perturbation theory. Certain semi-analytic features (i.e., Taylor-series nature) of our parallel RPM computations might be, perhaps, also re-classified as bringing an independent new insight into the structure of the spectra and, in particular, of the wave functions. 


\section{Appendix A: Two-parametric family of regularized singular interactions: phenomenological aspects.}

One of the key merits of the bound-state Schrödinger equations of the ordinary differential form

$$
\left[-\frac{d^{2}}{d x^{2}}+V(x)\right] \psi_{n}(x)=E_{n} \psi_{n}(x), \quad n=0,1, \ldots
$$

is that they combine a broad phenomenological applicability and methodical appeal with the formal friendliness of the linear differential equations of the second order. This has been re-emphasized in Ref. [16] where a judiciously chosen next-to-harmonic toy-model potential was studied in a specific strong-repulsion dynamical regime in which $g \gg 1$. In a continuation and generalization of this analysis (cf. section 4 here) one introduces a coordinate-dependent generalization of the couplings,

$$
V(x)=V_{\alpha, \beta, g}(x)=x^{2} \mu_{\alpha}(x)+\frac{g^{2} \nu_{\beta}(x)}{x^{6}} .
$$

Both of the additional non-constant functions of $x$ possess the same one-parametric power-law forms of $\mu_{\alpha}(x)=(i x)^{\alpha}, \alpha \geq 0$ and $\nu_{\beta}(x)=1 /(i x)^{\beta}, \beta \geq 0$.

This made the shape of the potential more flexible. Moreover, one may return to the original potential via an elementary limiting transition $\alpha \rightarrow 0$ and $\beta \rightarrow 0$. Far from this limit, on the contrary, the shape of the function(s) $\mu_{\alpha}(x)$ and $\nu_{\beta}(x)$ may be adapted more easily to phenomenological needs.

The list of formal reasons for our choice further incorporates also the quasisolvable nature of similar forces (cf. the fifth item in Table Nr. 1 of Ref. [21]), i.e., the feature which was made popular in monograph [22]) or the tractability of at least some of the related eigenvalue problems using continued fractions [23] or a specific simplicity of the asymptotic estimates of wave functions [24].

It makes sense to add that the studies of non-Hermitian but real-spectra-exhibiting quantum models may be perceived as one of the most dynamical branches of development of quantum theory after 1998 (see, e.g., reviews [14, 15, 25]). One of the fairly productive subbranches of these efforts was devoted to the mathematical idea (which may be dated back to the early nineties [5, 26]) that the spectrum of bound

states may be in fact controlled and modified by the mere ad hoc redefinition of the integration path of $x \in \mathcal{S} \subset \mathbb{C}$ (cf., e.g., [27]).

A consequent further extension of the latter mathematical idea (related closely to the presence of the strong singularities in $V(x)$ but getting us already beyond the limits of our present considerations) may be based on the question of what happens 
when the localization of the underlying integration path $\mathcal{S}$ is allowed to leave the plain complex plane (endowed, possibly, with a cut oriented upwards). In this direction it has been proposed [28] that in the definition of the integration path $\mathcal{S}$ one may and should try to replace the (cut) complex plane $\mathbb{C}$ by a more general Riemann surface $\mathcal{R}$. In the latter scenario (cf. also [29, 30]) one treats the general Riemann surface $\mathcal{R}$ as composed, in usual manner, of a set of individual Riemann-sheet cut planes, $\mathcal{R}=\bigcup_{j} \mathcal{R}_{j}$ where $\mathcal{R}_{j} \sim \mathbb{C}$. Then the path $\mathcal{S}$ of integration may and should encircle the branch-point singularities of $\mathcal{R}$, giving rise to several alternative, nonequivalent quantum systems living on the respective "tobogganic" complex curves. Thus, every such a system is described not only by the ordinary differential equation but also by the topologically nontrivial tobogganic path $\mathcal{S}$ (connecting, in general, several individual Riemann sheets) and, in addition, by a suitable definition of inner product in the underlying sophisticated physical Hilbert space $\mathcal{H}^{(S)}$ (cf. 29] for a deeper discussion of the latter point in tobogganic context). 


\section{References}

[1] F. G. Scholtz, H. B. Geyer and F. J. W. Hahne, Ann. Phys. (NY) 213 (1992) 74.

[2] M. Znojil, SIGMA 5 (2009) 001, arXiv: 0901.0700.

[3] F. J. Dyson, Phys. Rev. 102 (1956) 1217.

[4] A. Mostafazadeh, Class Quantum Grav 20 (2003) 155.

[5] V. Buslaev and V. Grecchi, J. Phys. A: Math. Gen. 26 (1993) 5541.

[6] H. F. Jones and J. Mateo, Phys. Rev. D 73 (2006) 085002.

[7] M. Znojil, Phys. Lett. A 259 (1999) 220.

[8] D. Bessis, private communication (1992).

[9] C. M. Bender and S. Boettcher, Phys. Rev. Lett. 80 (1998) 5243.

[10] P. Dorey, C. Dunning, R. Tateo, J. Phys. A: Math. Gen. 34 (2001) 5679.

[11] A. Mostafazadeh, J. Phys. A: Math. Gen. 39 (2006) 10171.

[12] A. Mostafazadeh and A. Batal, J. Phys. A: Math. Gen. 37 (2004) 11645.

[13] H. F. Jones, Phys. Rev. D 76 (2007) 125003.

[14] C. M. Bender, Rep. Prog. Phys. 70 (2007) 947.

[15] A. Mostafazadeh, Int. J. Geom. Meth. Mod. Phys. 7 (2010) 1191.

[16] M. Znojil, Int. J. Theor. Phys. 53 (2014) 2549.

[17] N. E. J. Bjerrum-Bohr, J. Math. Phys. 41 (2000) 2515.

[18] F. M. Fernández, Q. Ma and R. H. Tipping, Phys. Rev. A 39 (1989) 1605;

F. M. Fernández, Q. Ma, and R. H. Tipping, Phys. Rev. A 40 (1989) 6149.

[19] K. C. Shin, J. Math. Phys. 42 (2001) 2513.

[20] M. Znojil, F. Gemperle and O. Mustafa, J. Phys. A: Math. Gen. 35 (2002) 5781.

[21] M. Znojil, J. Phys. A: Math. Gen. 15 (1982) 2111. 
[22] A. G. Ushveridze, Quasi-Exactly Solvable Models in Quantum Mechanics. (IOP, Bristol, 1994).

[23] M. Znojil, J. Math. Phys. 31 (1990) 108.

[24] M. Znojil, Phys. Lett. A 158 (1991) 436.

[25] P. Dorey, C. Dunning and R. Tateo, J. Phys. A: Math. Theor. 40 (2007) R205.

[26] C. M. Bender and A. Turbiner, Phys. Lett. A 173 (1993) 442.

[27] C. M. Bender, S. Boettcher, H. F. Jones and V. M. Savage, J. Phys. A: Math. Gen. 32 (1999) 6771.

[28] M. Znojil, Phys. Lett. A 342 (2005) 36.

[29] M. Znojil, J. Phys. A: Math. Theor. 41 (2008) 215304.

[30] C. M. Bender, D. W. Hook, and S. P. Klevansky, J. Phys. A: Math. Theor. 45 (2012) 444003. 